\title{
Evaluation of Cellulolytic and Hemicellulolytic Abilities of Fungi Isolated from Coffee Residue and Sawdust Composts
}

\author{
MOHAMED FATHALlah EIDA ${ }^{1,2}$, TOShINORI NAGAOKA ${ }^{1}$, Jun WASAKI ${ }^{1}$, and KenJI KOUNO ${ }^{1 *}$ \\ ${ }^{1}$ Graduate School of Biosphere Science, Hiroshima University 4-4 Kagamiyama 1-Chome, Higashi-hiroshima 739-8528, \\ Japan; and ${ }^{2}$ Agricultural Microbiology Department, National Research Center 33 El Behous St., Dokki, 12622 Cairo, Egypt
}

(Received December 14, 2010—Accepted April 20, 2011—Published online May 11, 2011)

This study focused on the evaluation of cellulolytic and hemicellulolytic fungi isolated from sawdust compost (SDC) and coffee residue compost (CRC). To identify fungal isolates, the ITS region of fungal rRNA was amplified and sequenced. To evaluate enzyme production, isolates were inoculated onto wheat bran agar plates, and enzymes were extracted and tested for cellulase, xylanase, $\beta$-glucanase, mannanase, and protease activities using different azurine cross-linked (AZCL) substrates. In total, 18 isolates from SDC and 29 isolates from CRC were identified and evaluated. Four genera (Aspergillus, Galactomyces, Mucor, and Penicillium) and five genera (Aspergillus, Coniochaeta, Fusarium, Penicillium, and Trichoderma/Hypocrea) were dominant in SDC and CRC, respectively. Penicillium sp., Trichoderma sp., and Aspergillus sp. displayed high cellulolytic and hemicellulolytic activities, while Mucor isolates exhibited the highest $\beta$-glucanase and mannanase activities. The enzyme analyses revealed that Penicillium, Aspergillus, and Mucor isolates significantly contributed to the degradation of SDC, whereas Penicillium, Aspergillus, and Trichoderma isolates had a dominant role in the degradation of CRC. Notably, isolates SDCF5 (P. crustosum), CRCF6 (P. verruculosum), and $\mathrm{CRCF} 2$ and CRCF16 $(T$. harzianum/H. lixii) displayed high activity regarding cellulose and hemicellulose degradation, which indicates that these species could be beneficial for the improvement of biodegradation processes involving lignocellulosic materials.

Key words: fungi, cellulolytic activity, hemicellulolytic activity, AZCL-substrates, compost

Composting organic wastes represents an important pathway for carbon flow and nutrient cycling in both developed and developing countries. However, knowledge of the microbiology of this process is limited, particularly concerning the fungi associated with degradation of organic materials $(11,43)$.

Lignocellulosic materials, which are the major constituent of plant matter produced by photosynthesis, represent the most abundant renewable organic resource. These materials are composed of three types of polymers, namely cellulose, hemicellulose, and lignin, which are strongly engaged and chemically bonded $(25,38,58)$. For example, lignocellulosic materials of coffee residue are composed of $35 \%$ cellulose, $46.3 \%$ hemicellulose, and $18.8 \%$ lignin (42), whereas those of sawdust consist of $40-50 \%$ cellulose, $25-35 \%$ hemicellulose, and 20-30\% lignin (45). Due to their complexity, time-consuming processes are required to degrade these polymers and complete compost maturation.

Numerous microorganisms are capable of decomposing cellulose, although only a few of these microorganisms produce significant quantities of extracellular enzymes to decompose cellulose in vitro (47). Fungi are the main cellulase-producing microorganisms, but a few species of bacteria and actinomycetes have also been reported to yield cellulase $(47,56)$. Fungi, which are capable of degrading a wide variety of materials and compounds, are among the major decomposers of plant polymers, including lignocellulosic materials, in any ecosystem. Fungi can degrade

\footnotetext{
* Corresponding author. E-mail: kkouno@hiroshima-u.ac.jp; Tel: +81-82-424-7966; Fax: +81-82-424-0791.
}

mixtures of heterogeneous substrates, such as municipal solid waste, cattle manure, and agricultural and industrial wastes $(15,46)$.

Cellulolytic and hemicellulolytic enzymes play vital roles in biodegradation processes by fungi, bacteria, actinomycetes, and protozoa $(13,36)$. The efficient degradation of cellulolytic and hemicellulolytic plant materials requires a complex set of extracellular enzymes. All organisms known to efficiently degrade cellulose and hemicelluloses produce different degradative enzymes of varying specificities, which act synergistically $(9,54)$. For example, extracellular hydrolases and oxidoreductases produced by many microorganisms are involved in the degradation of lignocelluloses (53). Cellulose is the major polysaccharide in plant biomass (7), whereas xylans, $\beta$-glucans, and mannans are the most important constituents of hemicelluloses in plant cell walls (14). Consequently, determination of cellulase, xylanase, $\beta$-glucanase, and mannanase activities may indicate the cellulolytic and hemicellulolytic degradative potential of an organism.

Cellulolytic fungi are of great importance in our ecosystem, as they catalyze the enzymatic decay of lignocellulosic material. Notably, extracellular cellulases are produced at high levels by several fungi, including Trichoderma, Penicillium, Aspergillus, and Fusarium (19, 29). Filamentous fungi play critical roles in the biodegradation of numerous types of organic waste materials by releasing exoenzymes involved in oxidation and hydrolysis (15). Therefore, the study of fungal biodegradation processes must include determination of an organism's catalytic capability.

The application of screening techniques that are rapid and easy to evaluate allows the testing of a large number of 
isolates for the selection of highly active strains (24). However, the qualitative and semi-quantitative screening of microorganisms which are able to produce cellulases and xylanases using classical screening methods does not typically provide conclusive results due to difficulties in the evaluation of substrate consumption during cultivation on agar media with cellulose or xylan (20). For the detection of highly active strains during primary screening, a few recent studies have described effective methods based on the utilization of insoluble chromogenic substrates that provide results with greater fidelity (21). A number of different plate screening methods to identify polysaccharide-degrading microorganisms have been described (49), which are typically based on either the formation of complexes between polysaccharides and dyes, the solubility and gel-forming characteristics of polysaccharides, or the use of soluble and/or insoluble dye-labeled polysaccharides $(50,58)$. During hydrolysis, cross-linked macromolecular substrates labeled with colorant particles are attacked, resulting in the solubilization of the colorant particles and formation of a colored zone around microorganisms which are able to produce extracellular enzymes. The intensity of the colored zone depends on several factors, including substrate and enzyme concentrations, and the catalytic properties of enzymes (16). As chromogenic substrates, commercially available insoluble azurine cross-linked (AZCL) substrates, which contain a blue azurine copper compound bound to the target polysaccharide, have proven effective for these assays (37). Using these substrates, a clear correlation between the diameters of the formed blue haloes and levels of enzymes produced was demonstrated (50).

Traditional fungal identification methods are technically difficult and time consuming. Recently, several improved techniques that use DNA sequencing for microbial identification have been developed (30). For example, variations in ribosomal-RNA (rRNA) internal transcribed spacer (ITS) regions have been used for the molecular identification of fungi to the species level. The non-coding ITS region is suitable for fungal identification because of its high copy number in fungal genomes and fast rate of evolution, which results in high sequence variation among even closely related species $(27,32)$. Therefore, DNA sequences in the ITS region generally provide superior taxonomic characters than those from coding regions $(5,31)$.

In this study, sawdust compost (SDC) and coffee residue compost (CRC) were selected for the isolation and characterization of cellulolytic and hemicellulolytic fungi due to their high cellulose and hemicellulose content, and also their low degradation rates. In addition, most previous papers have only examined limited enzymatic activities of fungal groups, and we were unable to find any reports describing multiple activities of different fungal groups isolated from compost.
Therefore, several fungi were isolated from SDC and CRC, identified, and screened for cellulase, xylanase, $\beta$-glucanase, mannanase, and protease activities.

\section{Materials and Methods}

\section{Fungal isolation}

The SDC produced from residue of a culture medium for mushroom was obtained from the Agricultural Cooperative Association of Saitama prefecture, while CRC was collected from a composting center located in Hiroshima prefecture. Basically, they were produced by composting heaps that were turned periodically during 2-3 months until they reached maturity. The chemical and biological properties of SDC and CRC are presented in Table 1.

Fungi were isolated from SDC and CRC using a dilution plate method. The primary suspensions were prepared by suspending $10 \mathrm{~g}$ of each compost type in $90 \mathrm{~mL}$ of sterile distilled water and were shaken $(150 \mathrm{rpm})$ for $30 \mathrm{~min}$ at room temperature. Then, ten-fold serial dilutions were prepared in sterilized distilled water. One hundred microliters of the $10^{-5}$ dilution was spread on Martin's medium (glucose, $10 \mathrm{~g} \mathrm{~L}^{-1}$; peptone, $5 \mathrm{~g} \mathrm{~L}^{-1} ; \mathrm{KH}_{2} \mathrm{PO}_{4}, 1 \mathrm{~g} \mathrm{~L}^{-1}$; $\mathrm{MgSO}_{4} \cdot 7 \mathrm{H}_{2} \mathrm{O}, 0.5 \mathrm{~g} \mathrm{~L}^{-1}$; Rose Bengal, $0.033 \mathrm{~g} \mathrm{~L}^{-1}$; and streptomycin, $0.03 \mathrm{~g} \mathrm{~L}^{-1} ; \mathrm{pH} 5.5-6.0$ ) and plates were then incubated at $30^{\circ} \mathrm{C}$ for 5-7 days. Five plates were prepared for each compost type. All the colonies of the most typical plate were selected. The colonies were transferred several times on the same medium and further incubated at $30^{\circ} \mathrm{C}$ for 7 days to obtain pure cultures. All fungal isolates were maintained on potato dextrose agar (PDA, Nissui Pharmaceutical, Tokyo, Japan) slants at $4^{\circ} \mathrm{C}$. Fungal isolates were identified conventionally according to their morphological features to determine their genera $(2,8,17,18,52)$.

\section{Enzyme preparation}

For enzyme production, all fungal isolates were inoculated onto wheat bran agar (WBA) plates (wheat bran, $30 \mathrm{~g} \mathrm{~L}^{-1}$; agar, 15 $\mathrm{g} \mathrm{L}^{-1} ; \mathrm{ZnSO}_{4} \cdot 7 \mathrm{H}_{2} \mathrm{O}, 0.01 \mathrm{~g} \mathrm{~L}^{-1}$; and $\mathrm{CuSO}_{4} \cdot 5 \mathrm{H}_{2} \mathrm{O}, 0.005 \mathrm{~g} \mathrm{~L}^{-1}$; $\mathrm{pH}$ 7.4) (37). The plates were then incubated for 7 days at $25^{\circ} \mathrm{C}$ in darkness in perforated plastic bags.

Enzyme complexes of the isolated fungi were extracted following the method described by Pedersen et al. (37). Briefly, enzymes were extracted from 7-day-old cultures grown on WBA plates. Twenty agar plugs $(6 \mathrm{~mm}$ in diameter) with mycelia were excised from the plate of each isolate. The plugs were transferred to a $14-\mathrm{mL}$ vial, $2.5 \mathrm{~mL}$ of distilled water was added, and the vial was then shaken at $150 \mathrm{rpm}$ for $2 \mathrm{~h}$ on a Yamato SA-31 shaker (Yamato Scientific, Tokyo, Japan) at room temperature. The extracted solution was filtered through a sterile $0.45-\mu \mathrm{m}$ membrane filter ( 25 $\mathrm{mm}$ in diameter, Toyo Roshi, Tokyo, Japan). The extracted enzyme solutions were kept at $-30^{\circ} \mathrm{C}$ until used.

\section{Enzyme assays}

Five different AZCL substrates (AZCL-HE-cellulose, -arabinoxylan, -barley $\beta$-glucan, -galactomannan, and -casein [Megazyme, Bray, Ireland]) were used for enzyme assays. Assays were performed according to the method described by Pedersen et al. (37). Briefly, after the assay suspension containing agarose and each AZCL substrate was solidified in Petri dishes, 15 wells with a 6-mm diameter were made in the agarose plates using a cork borer, and $15 \mu \mathrm{L}$ of enzyme extract was added to each well. The plates were

Table 1. Chemical and biological properties of SDC and CRC

\begin{tabular}{|c|c|c|c|c|c|c|}
\hline \multirow{2}{*}{ Compost used } & \multirow{2}{*}{ Dry matter $\left(\mathrm{g} \mathrm{kg}^{-1}\right)$} & \multicolumn{3}{|c|}{ Total $\left(\mathrm{g} \mathrm{kg}^{-1}\right)^{*}$} & \multirow{2}{*}{$\mathrm{C} / \mathrm{N}$ ratio } & \multirow{2}{*}{$\begin{array}{l}\text { Fungal population } \\
\left(\times 10^{6} \mathrm{cfu} \mathrm{g}^{-1}\right)^{*}\end{array}$} \\
\hline & & $\mathrm{C}$ & $\mathrm{N}$ & $\mathrm{P}$ & & \\
\hline SDC & 538 & 456 & 14.7 & 12.3 & 30.0 & 2.3 \\
\hline $\mathrm{CRC}$ & 390 & 448 & 21.4 & 0.7 & 20.9 & 3.0 \\
\hline
\end{tabular}

* Values expressed on an oven-dry basis. 
incubated for $24 \mathrm{~h}$ at $30^{\circ} \mathrm{C}$ in the case of HE-cellulose, while for all other substrates the incubation time was 6 h. Enzymatic activities were determined by measuring the diameter of the blue zone formed around each well, which was then converted to area $\left(\mathrm{mm}^{2}\right)$ and subtracted from the area of the well.

\section{DNA extraction and PCR amplification}

For DNA extraction, all fungal isolates were grown using PDA medium and incubated at $30^{\circ} \mathrm{C}$ for 7 days. DNA was then extracted with an Isoplant Kit (Nippon Gene, Toyama, Japan) according to the manufacturer's instructions.

PCR was performed using a GeneAmp PCR System 2700 (Applied Biosystems, Foster City, CA, USA). ITS regions of rRNA were amplified using ITS1 (TCCGTAGGTGAACCTGCGG) and ITS4 (TCCTCCGCTTATTGATATGC) as forward and reverse primers, respectively, according to White et al. (57). All reactions were carried out in $50-\mu \mathrm{L}$ volumes containing genomic DNA (1.0 $\mu \mathrm{L} 20 \mathrm{ng} \mu \mathrm{L}^{-1}$ ), $5.0 \mu \mathrm{L}$ of $10 \times$ Buffer (including $20 \mathrm{mM} \mathrm{MgCl}{ }_{2}$ ), $5.0 \mu \mathrm{L}$ of dNTP mixture (2.5 mM each), $2.5 \mathrm{U}$ of Ex Taq (Takara Bio, Otsu, Japan) and $1.0 \mu \mathrm{L}(20 \mu \mathrm{M})$ of each primer (ITS1 and ITS4). The thermal cycling program was as follows: $94^{\circ} \mathrm{C}$ for 2 min, followed by 35 cycles of denaturation at $94^{\circ} \mathrm{C}$ for $10 \mathrm{~s}$, annealing at $63^{\circ} \mathrm{C}$ for $1 \mathrm{~min}$, and extension at $72^{\circ} \mathrm{C}$ for $1 \mathrm{~min}$, with a final extension step at $72^{\circ} \mathrm{C}$ for $5 \mathrm{~min}$. The annealing temperature was increased to $65^{\circ} \mathrm{C}$ to obtain a single band from isolates SDCF1, SDCF10, SDCF17, and CRCF27. The PCR products were analyzed by agarose gel electrophoresis, stained with ethidium bromide, and visualized by UV transillumination with an AE-6932GXCF printgraph (Atto, Tokyo, Japan). The amplified PCR products were purified using a QIAquick PCR purification Kit (Qiagen, Hilden, Germany) according to the protocol provided by the manufacturer.

\section{Sequencing and data analysis}

Sequencing of the amplified ITS regions of the fungal rRNA was performed using a BigDyeR Terminator v3.1 Cycle Sequencing Kit (Applied Biosystems, Carlsbad, CA, USA) on an Applied Biosystems 3730xl DNA Analyzer. Similarities of the determined fungal DNA sequences with other known species were investigated by comparisons with sequence data in the National Center for Biotechnology Information (NCBI) database using the BLASTN 2.2.24 program (59). A phylogenetic tree based on the ITS regions of partial rRNA sequences was constructed using the neighborjoining method contained within the Clustal X program (51) and MEGA4 software (48).

Nucleotide sequence accession numbers

The nucleotide sequences determined in this study have been deposited with GenBank under the nucleotide accession numbers HQ657273-HQ657319.

\section{Results}

\section{Fungal isolation from compost}

Two compost types, SDC and CRC, were subjected to screening for the isolation and characterization of cellulolytic and hemicellulolytic fungi. A total of 18 isolates were obtained from SDC, while 29 were isolated from CRC (Table 2).

\section{Identification and phylogenetic analysis of fungal isolates}

The morphological characteristics of the fungi showed that the isolates were species of the genera Mucor, Trichoderma, Penicillium, Coniochaeta, Galactomyces (Geotrichum), Aspergillus, and Fusarium.

Although the analysis of fungal ITS sequences revealed a wide diversity of fungal taxa in both SDC and CRC, BLAST searches demonstrated that four genera (Mucor, Penicillium, Galactomyces, and Aspergillus) were dominant in SDC (Table 2 and Fig. 1). The phylogenetic tree constructed based on ITS sequences of SDC isolates indicated that nine isolates were close to $M$. circinelloides with 99-100\% similarity, while five isolates appeared to be members of the genus Penicillium, as they shared $100 \%$ similarity with $P$. crustosum. In addition, three isolates were close (98$100 \%$ similarity) to $G$. geotrichum, whereas one isolate (SDCF16) shared $100 \%$ similarity with A. fumigatus, according to BLAST searches of sequences in the NCBI database, as summarized in Table 2 .

For the fungal isolates from $\mathrm{CRC}$, data derived from BLAST analyses and the phylogenetic tree of ITS sequences revealed that five genera (Aspergillus, Penicillium, Coniochaeta, Fusarium and Trichoderma/Hypocrea) were dominant, with similarities ranging from 98 to $100 \%$ (Fig. 2). Among the 29 isolates in total, 14 were closely related to the genus Trichoderma/Hypocrea, with three of these isolates matching $H$. virens (T. virens) with 99-100\% maximum identity, and the other 11 displaying 99-100\% identity with $H$. lixii ( $T$. harzianum). Eleven isolates were matched with two different species of the genus Coniochaeta; nine of these displayed $99 \%$ similarity to $C$. ligniaria, while two isolates were closely related to $C$. velutina $(98 \%$ similarity). In addition, two isolates (CRCF6 and CRCF12) exhibited $99 \%$ similarity with $P$. verruculosum, while CRCF11 and CRCF27 matched 100\% with A. fumigatus and F. acuminatum, respectively (Table 2).

\section{Characterization of SDC fungal isolates}

In SDC compost, Mucor sp. was the most dominant fungal genus, representing $50 \%(9 / 18)$ of isolates. Although the Mucor isolates all showed higher $\beta$-glucanase and mannanase activities than the other SDC isolates, they did not produce cellulase or xylanase when grown on WBA medium. In addition, only three Mucor isolates (SDCF19, SDCF21, and

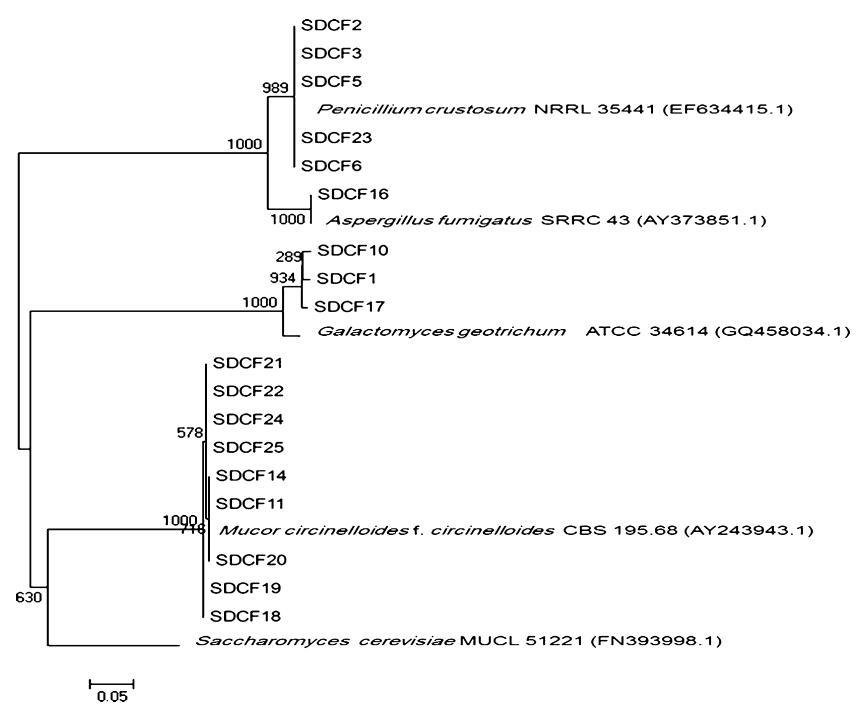

Fig. 1. Neighbor-joining tree showing the relationship between ITS sequences from fungi isolated from sawdust compost (SDC). Bootstrap values of the neighbor-joining analysis with 1,000 replications are shown on the branches. The scale bar represents the number of changes per nucleotide position (substitution/site). 
Table 2. Top BLAST results for the ITS region of fungal rRNA and GenBank sequence accession numbers of fungi isolated from SDC and CRC

\begin{tabular}{|c|c|c|c|}
\hline Isolate No. & GenBank accession No. & Top BLAST hit GenBank & Percentage of similarity \\
\hline SDCF1 & HQ657273 & Galactomyces geotrichum (DQ907937.1) & 100 \\
\hline SDCF2 & HQ657274 & Penicillium crustosum (FJ228197.1) & 100 \\
\hline SDCF3 & HQ657275 & Penicillium crustosum (EF634415.1) & 100 \\
\hline SDCF5 & HQ657276 & Penicillium crustosum (HM037943.1) & 100 \\
\hline SDCF6 & HQ657277 & Penicillium crustosum (FJ228197.1) & 100 \\
\hline SDCF10 & HQ657278 & Galactomyces geotrichum (DQ681363.2) & 98.8 \\
\hline SDCF11 & HQ657279 & Mucor circinelloides (DQ118991.1) & 100 \\
\hline SDCF14 & HQ657280 & Mucor circinelloides f. circinelloides (DQ118991.1) & 100 \\
\hline SDCF16 & HQ657281 & Aspergillus fumigatus (EF134624.1) & 100 \\
\hline SDCF17 & HQ657282 & Galactomyces geotrichum (DQ907937.1) & 99.1 \\
\hline SDCF18 & HQ657283 & Mucor circinelloides (DQ118989.1) & 99.8 \\
\hline SDCF19 & HQ657284 & Mucor circinelloides (DQ118989.1) & 100 \\
\hline SDCF20 & HQ657285 & Mucor circinelloides f. circinelloides (EU484236.1) & 100 \\
\hline SDCF21 & HQ657286 & Mucor circinelloides (AY213658.1) & 100 \\
\hline SDCF22 & HQ657287 & Mucor circinelloides (AY213658.1) & 99.8 \\
\hline SDCF23 & HQ657288 & Penicillium crustosum (FJ228197.1) & 100 \\
\hline SDCF24 & HQ657289 & Mucor circinelloides f. circinelloides (EU484236.1) & 99.8 \\
\hline SDCF25 & HQ657290 & Mucor circinelloides f. circinelloides (EU484236.1) & 99.8 \\
\hline CRCF1 & HQ657291 & Coniochaeta ligniaria (AY198390.1) & 99.8 \\
\hline CRCF2 & HQ657292 & Hypocrea lixii (HM176572.1) & 100 \\
\hline CRCF3 & HQ657293 & Hypocrea lixii (HM176572.1) & 100 \\
\hline CRCF4 & HQ657294 & Hypocrea virens (GU066623.1) & 100 \\
\hline CRCF5 & HQ657295 & Hypocrea virens (GU066623.1) & 100 \\
\hline CRCF6 & HQ657296 & Penicillium verruculosum (HM049911.1) & 99.2 \\
\hline CRCF7 & HQ657297 & Coniochaeta ligniaria (AY198390.1) & 99.8 \\
\hline CRCF8 & HQ657298 & Hypocrea lixii (HM176572.1) & 100 \\
\hline CRCF9 & HQ657299 & Hypocrea lixii (HM176572.1) & 100 \\
\hline CRCF10 & HQ657300 & Hypocrea lixii (HM176572.1) & 100 \\
\hline CRCF11 & HQ657301 & Aspergillus fumigatus (EF134624.1) & 100 \\
\hline CRCF12 & HQ657302 & Penicillium verruculosum (HM049911.1) & 99.6 \\
\hline CRCF13 & HQ657303 & Hypocrea lixii (HM176572.1) & 100 \\
\hline CRCF14 & HQ657304 & Hypocrea lixii (FJ517550.1) & 99.6 \\
\hline CRCF15 & HQ657305 & Hypocrea lixii (FJ442646.1) & 99.8 \\
\hline CRCF16 & HQ657306 & Hypocrea lixii (HM176572.1) & 99.8 \\
\hline CRCF17 & HQ657307 & Coniochaeta ligniaria (AY198390.1) & 99.8 \\
\hline CRCF18 & HQ657308 & Hypocrea lixii (HM176572.1) & 100 \\
\hline CRCF20 & HQ657309 & Coniochaeta ligniaria (AY198390.1) & 99.8 \\
\hline CRCF21 & HQ657310 & Coniochaeta ligniaria (AY198390.1) & 99.8 \\
\hline CRCF22 & HQ657311 & Coniochaeta ligniaria (AY198390.1) & 99.8 \\
\hline CRCF23 & HQ657312 & Coniochaeta ligniaria (AY198390.1) & 99.8 \\
\hline CRCF24 & HQ657313 & Coniochaeta velutina (GQ154545.1) & 98.9 \\
\hline CRCF26 & HQ657314 & Hypocrea virens (GU066623.1) & 99.8 \\
\hline CRCF27 & HQ657315 & Fusarium acuminatum (HM068325.1) & 100 \\
\hline CRCF28 & HQ657316 & Hypocrea lixii (HM176572.1) & 99.7 \\
\hline CRCF29 & HQ657317 & Coniochaeta ligniaria (AY198390.1) & 99.8 \\
\hline CRCF30 & HQ657318 & Coniochaeta velutina (GQ154545.1) & 98.5 \\
\hline CRCF31 & HQ657319 & Coniochaeta ligniaria (AY198390.1) & 99.8 \\
\hline
\end{tabular}

SDCF indicates fungi that were isolated from sawdust compost, and CRCF indicates fungi that were isolated from coffee residue compost, while the numbers represent the isolate number.

SDCF22) produced protease (Table 3).

The five isolates identified as $P$. crustosum $(100 \%$ similarity) possessed cellulase, xylanase, $\beta$-glucanase, and mannanase activities, and did not exhibit protease activity, with the exception of SDCF6, which produced proteases, but not cellulases. Among the SDC isolates, Penicillium isolates displayed the highest xylanase activity and relatively high cellulase activity. Notably, SDCF5 exhibited the highest cellulase activity and comparatively high xylanase activity among the Penicillium isolates (Table 3).

The three Galactomyces isolates did not show any activities towards the different AZCL substrates. Finally, the single isolate identified as A. fumigatus (SDCF16) exhibited positive activity in all assays. SDCF16 showed the highest cellulase activity among SDC isolates, in addition to relatively high $\beta$-glucanase and mannanase activities (Table 3 ).

\section{Characterization of CRC fungal isolates}

Members of the genus Trichoderma/Hypocrea were the most abundant fungi isolated from CRC (14/29), with the 14 identified isolates belonging to only one of two species, either $T$. harzianum/H. lixii or T. virens. All Trichoderma 


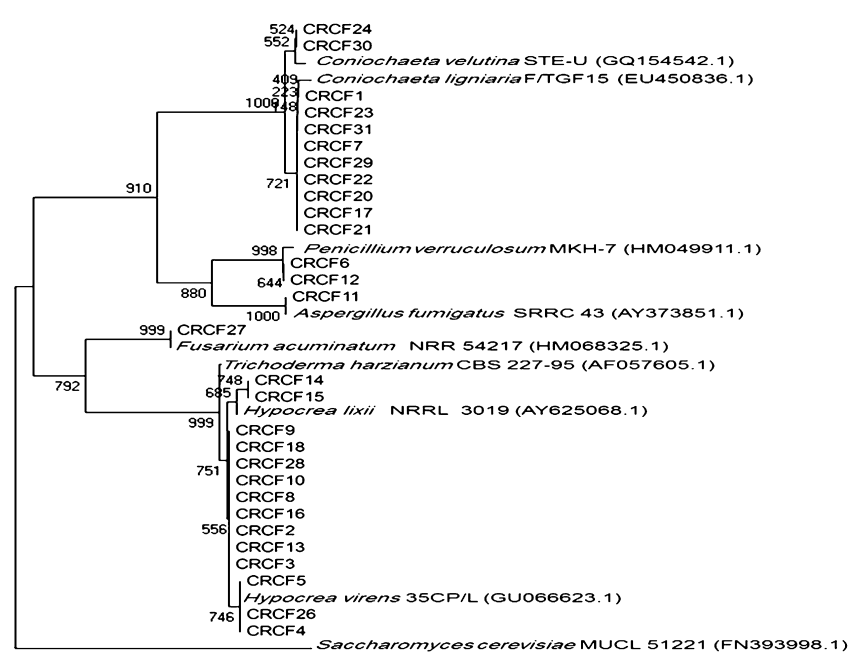

$\stackrel{\bullet}{0.02}$

Fig. 2. Neighbor-joining tree showing the relationship between ITS sequences from fungi isolated from coffee residue compost (CRC). Bootstrap values of the neighbor-joining analysis with 1,000 replications are shown on the branches. The scale bar represents the number of changes per nucleotide position (substitution/site).

isolates, with the exception of CRCF3, CRCF5, and CRCF15, exhibited cellulase activity. CRCF2 and CRCF16 (T. harzianum/H. lixii) produced the highest cellulase activity among Trichoderma isolates. The xylanase activity of Trichoderma isolates exhibited a similar trend with cellulase activity (Table 4), as all isolates except CRCF15 had xylanase activity. $T$. virens isolates (CRCF4, CRCF5, and CRCF26) did not possess $\beta$-glucanase activity, while $T$. harzianum isolates displayed the highest $\beta$-glucanase activity among $\mathrm{CRC}$ isolates. In addition, while all isolates except CRCF15 had mannanase activity, only three isolates (CRCF14, CRCF15 and CRCF16) could degrade AZCL-casein as a substrate in the protease assay (Table 4).

Coniochaeta sp. was the second most abundant genus in CRC (11/29); however, only one isolate (CRCF23) showed cellulase activity, which was the lowest activity among the $\mathrm{CRC}$ isolates. Seven Coniochaeta isolates produced xylanase, with CRCF2 1 having the highest activity among Coniochaeta isolates. All Coniochaeta isolates displayed $\beta$-glucanase, mannanase, and protease activities, with the exception of CRCF24 and CRCF30, which did not possess mannanase activity (Table 4).

Notably, $P$. verruculosum (CRCF6) showed the highest cellulase activity of all CRC isolates, while the other $P$. verruculosum isolate (CRCF12) did not produce cellulase under the assay conditions. Both isolates exhibited xylanase, $\beta$-glucanase, and mannanase activities, although no protease activity was detected. In addition to high cellulase activity, CRCF6 showed moderately high $\beta$-glucanase and mannanase activities (Table 4). Similar to isolate SDCF16, A. fumigatus (CRCF11) displayed cellulase, xylanase, $\beta$-glucanase, mannanase, and protease activities, while the $F$. acuminatum isolate (CRCF27) showed only relatively low xylanase and $\beta$-glucanase activities (Table 4).

\section{Discussion}

In this study, we investigated the fungi present in SDC and CRC in an attempt to clarify the role of isolated fungal groups in the degradation of lignocellulosic materials through the characterization of enzymatic activities. Although our results suggest that different fungi are associated with cellulolytic and hemicellulolytic activities in the two compost types, Penicillium and Aspergillus were dominant in both SDC and CRC. The variance in fungal community between SDC and CRC may reflect the differences in the raw material components available for composting such as differences in

Table 3. Enzymatic activities of fungi isolated from SDC

\begin{tabular}{|c|c|c|c|c|c|c|}
\hline \multirow{2}{*}{ Genus } & \multirow{2}{*}{ Isolate No. } & \multicolumn{5}{|c|}{ Area of the blue halo zone $\left(\mathrm{mm}^{2}\right)$} \\
\hline & & Cellulase & Xylanase & $\beta$-Glucanase & Mannanase & Protease \\
\hline \multirow{9}{*}{ Mucor } & SDCF11 & N.D. & N.D. & $287 \pm 27.1$ & $150 \pm 15.1$ & N.D. \\
\hline & SDCF14 & N.D. & N.D. & $301 \pm 35.5$ & $170 \pm 14.2$ & N.D. \\
\hline & SDCF18 & N.D. & N.D. & $285 \pm 30.3$ & $158 \pm 18.5$ & N.D. \\
\hline & SDCF19 & N.D. & N.D. & $270 \pm 23.7$ & $140 \pm 12.8$ & $20 \pm 11.6$ \\
\hline & SDCF20 & N.D. & N.D. & $303 \pm 25.1$ & $144 \pm 21.6$ & N.D. \\
\hline & SDCF21 & N.D. & N.D. & $275 \pm 22.2$ & $182 \pm 15.7$ & $59 \pm 8.4$ \\
\hline & SDCF22 & N.D. & N.D. & $286 \pm 26.3$ & $202 \pm 18.8$ & $36 \pm 9.0$ \\
\hline & SDCF24 & N.D. & N.D. & $212 \pm 39.4$ & $180 \pm 16.5$ & N.D. \\
\hline & SDCF25 & N.D. & N.D. & $323 \pm 27.1$ & $187 \pm 23.3$ & N.D. \\
\hline \multirow{5}{*}{ Penicillium } & SDCF2 & $44 \pm 15.7$ & $315 \pm 27.8$ & $151 \pm 14.4$ & $70 \pm 11.3$ & N.D. \\
\hline & SDCF3 & $60 \pm 19.1$ & $298 \pm 35.0$ & $162 \pm 16.7$ & $49 \pm 9.5$ & N.D. \\
\hline & SDCF5 & $94 \pm 19.0$ & $314 \pm 35.6$ & $153 \pm 15.4$ & $48 \pm 10.7$ & N.D. \\
\hline & SDCF6 & N.D. & $212 \pm 1.2$ & $215 \pm 22.1$ & $129 \pm 20.6$ & $45 \pm 9.4$ \\
\hline & SDCF23 & $73 \pm 17.7$ & $280 \pm 26.6$ & $115 \pm 15.5$ & $20 \pm 8.7$ & N.D. \\
\hline \multirow{3}{*}{ Galactomyces } & SDCF1 & N.D. & N.D. & N.D. & N.D. & N.D. \\
\hline & SDCF10 & N.D. & N.D. & N.D. & N.D. & N.D. \\
\hline & SDCF17 & N.D. & N.D. & N.D. & N.D. & N.D. \\
\hline Aspergillus & SDCF16 & $117 \pm 14.6$ & $99 \pm 11.9$ & $223 \pm 20.4$ & $147 \pm 15.0$ & $47 \pm 8.7$ \\
\hline
\end{tabular}

Enzymatic activities are reported as the area $\left(\mathrm{mm}^{2}\right)$ of the blue halo surrounding wells $( \pm \mathrm{SD})$ containing fungal enzyme extracts for five assays using AZCL-HE-cellulose (cellulase), AZCL-arabinoxylan (xylanase), AZCL-barley $\beta$-glucan ( $\beta$-glucanase), AZCL-galactomannan (mannanase), and AZCL-casein (protease) as substrates. The values in bold represent the isolate with the highest activity for each assay. N.D., not detected. 
Table 4. Enzymatic activities of fungi isolated from CRC

\begin{tabular}{|c|c|c|c|c|c|c|}
\hline \multirow{2}{*}{ Genus } & \multirow{2}{*}{ Isolate No. } & \multicolumn{5}{|c|}{ Area of the blue halo zone $\left(\mathrm{mm}^{2}\right)$} \\
\hline & & Cellulase & Xylanase & $\beta$-Glucanase & Mannanase & Protease \\
\hline \multirow{14}{*}{ Trichoderma/Hypocrea } & CRCF2 & $172 \pm 21.4$ & $172 \pm 20.5$ & $171 \pm 16.6$ & $110 \pm 11.8$ & N.D. \\
\hline & CRCF3 & N.D. & $156 \pm 29.1$ & $193 \pm 20.4$ & $95 \pm 11.1$ & N.D. \\
\hline & CRCF8 & $99 \pm 11.7$ & $196 \pm 18.0$ & $204 \pm 16.5$ & $131 \pm 13.9$ & N.D. \\
\hline & CRCF9 & $119 \pm 18.9$ & $194 \pm 17.6$ & $217 \pm 16.6$ & $146 \pm 11.7$ & N.D. \\
\hline & CRCF10 & $73 \pm 11.9$ & $192 \pm 22.6$ & $220 \pm 17.5$ & $110 \pm 13.0$ & N.D. \\
\hline & CRCF13 & $62 \pm 9.9$ & $174 \pm 19.2$ & $201 \pm 20.0$ & $124 \pm 10.4$ & N.D. \\
\hline & CRCF14 & $99 \pm 10.4$ & $255 \pm 22.6$ & $147 \pm 15.0$ & $142 \pm 20.4$ & $75 \pm 11.4$ \\
\hline & CRCF15 & N.D. & N.D. & $17 \pm 13.2$ & N.D. & $56 \pm 7.9$ \\
\hline & CFRCF16 & $131 \pm 21.3$ & $172 \pm 19.2$ & $205 \pm 18.3$ & $153 \pm 14.5$ & $36 \pm 10.2$ \\
\hline & CRCF18 & $67 \pm 12.6$ & $193 \pm 15.0$ & $189 \pm 16.2$ & $139 \pm 18.8$ & N.D. \\
\hline & CRCF28 & $128 \pm 18.8$ & $163 \pm 17.8$ & $188 \pm 18.9$ & $142 \pm 13.5$ & N.D. \\
\hline & CRCF4 & $77 \pm 13.4$ & $182 \pm 14.6$ & N.D. & $77 \pm 13.9$ & N.D. \\
\hline & CRCF5 & N.D. & $39 \pm 13.9$ & N.D. & $47 \pm 9.7$ & N.D. \\
\hline & CRCF26 & $73 \pm 13.7$ & $100 \pm 12.6$ & N.D. & $58 \pm 10.3$ & N.D. \\
\hline \multirow{11}{*}{ Coniochaeta } & CRCF1 & N.D. & $96 \pm 9.9$ & $79 \pm 10.0$ & $47 \pm 11.7$ & $31 \pm 14.4$ \\
\hline & CRCF7 & N.D. & $69 \pm 16.1$ & $51 \pm 9.2$ & $30 \pm 6.7$ & $29 \pm 12.3$ \\
\hline & CRCF17 & N.D. & N.D. & $21 \pm 10.6$ & $27 \pm 6.7$ & $38 \pm 7.1$ \\
\hline & CRCF20 & N.D. & $61 \pm 20.1$ & $104 \pm 15.1$ & $51 \pm 12.9$ & $49 \pm 7.0$ \\
\hline & CRCF21 & N.D. & $150 \pm 20.8$ & $61 \pm 9.2$ & $69 \pm 11.5$ & $38 \pm 6.1$ \\
\hline & CRCF22 & N.D. & N.D. & $78 \pm 10.2$ & $20 \pm 9.1$ & $26 \pm 9.2$ \\
\hline & CRCF23 & $39 \pm 13.8$ & $48 \pm 12.1$ & $120 \pm 15.1$ & $67 \pm 13.6$ & $46 \pm 8.1$ \\
\hline & CRCF29 & N.D. & $77 \pm 14.7$ & $119 \pm 13.9$ & $58 \pm 14.3$ & $62 \pm 7.4$ \\
\hline & CRCF31 & N.D. & $44 \pm 10.2$ & $47 \pm 11.1$ & $81 \pm 13.4$ & $23 \pm 9.6$ \\
\hline & CRCF24 & N.D. & N.D. & $95 \pm 11.4$ & N.D. & $26 \pm 9.0$ \\
\hline & CRCF30 & N.D. & N.D. & $183 \pm 18.6$ & N.D. & $60 \pm 12.2$ \\
\hline \multirow{2}{*}{ Penicillium } & CRCF6 & $194 \pm 16.1$ & $83 \pm 12.4$ & $182 \pm 17.2$ & $91 \pm 11.5$ & N.D. \\
\hline & CRCF12 & N.D. & $116 \pm 20.0$ & $170 \pm 18.3$ & $38 \pm 10.2$ & N.D. \\
\hline Aspergillus & CRCF11 & $116 \pm 13.3$ & $90 \pm 15.9$ & $201 \pm 18.7$ & $78 \pm 11.2$ & $69 \pm 10.0$ \\
\hline Fusarium & CRCF27 & N.D. & $41 \pm 13.4$ & $54 \pm 12.3$ & N.D. & N.D. \\
\hline
\end{tabular}

Enzymatic activities are reported as the area $\left(\mathrm{mm}^{2}\right)$ of the blue halo surrounding wells ( \pm SD) containing fungal enzyme extracts for five assays using AZCL-HE-cellulose (cellulase), AZCL-arabinoxylan (xylanase), AZCL-barley $\beta$-glucan ( $\beta$-glucanase), AZCL-galactomannan (mannanase), and AZCL-casein (protease) as substrates. The values in bold represent the isolate with the highest activity for each assay. N.D., not detected.

cellulose, hemicellulose, lignin, protein, ash, fibers, lipids, caffeine, and tannins (4, 6, 12, 35). Jing-Chun et al. (28) demonstrated that during composting, different raw materials are the main factors affecting microbial community structure. Our analyses revealed that a number of isolates, including $P$. crustosum (SDCF5), P. verruculosum (CRCF6), and $T$. harzianum/H. lixii (CRCF2 and CRCF16), possess high cellulolytic and hemicellulolytic activities, and likely play a dominant role in degrading lignocellulosic materials in compost.

The genera isolated from SDC were Mucor (9 isolates), Penicillium (5 isolates), Galactomyces (3 isolates) and Aspergillus (1 isolate), while Trichoderma/Hypocrea (14 isolates), Coniochaeta (11 isolates), Penicillium (2 isolates), Aspergillus (1 isolate), and Fusarium (1 isolate) were isolated from CRC. Our results are consistent with previous reports that have found that Aspergillus, Trichoderma, Penicillium, Mucor, and Fusarium fungi are commonly present in compost (4, 6, 36). In addition, Purnomo et al. (40) isolated $M$. circinelloides and G. geotrichum from cattle manure compost, while Hatamoto et al. (23) reported that Coniochaeta is a key eukaryote associated with the decomposition of rice straw compost in Japanese rice paddy fields. Sánchez (42) also reported the isolation of filamentous fungi species of
Penicillium, Trichoderma, Aspergillus, and Fusarium from lignocellulosic residues. Notably, our results showed that Penicillium and Aspergillus are dominant in both SDC and $\mathrm{CRC}$, a conclusion which is supported by several reports that have demonstrated the ubiquity of Aspergillus and Penicillium fungi in environmental samples, which are typically found as saprophytes in compost and soil $(4,6,10$, 39).

In this study, we evaluated cellulase, xylanase, $\beta$ glucanase, mannanase, and protease activities of several fungal groups isolated from compost. The enzymatic activities of the isolated fungi grown on wheat bran medium clearly varied not only between genera and species, but also between individual strains in each species. This result is in accordance with those of Pederson et al. (37), who found that enzymatic activities of isolates from the genus Ulocladium varied among species and also individual strains of the same species. In addition, they determined that the source of isolation affected enzyme production in individual strains.

Among the SDC isolates, G. geotrichum strains did not exhibit any activities towards cellulose, xylan, $\beta$-glucan, mannan, or casein. In contrast, M. circinelloides isolates showed the highest $\beta$-glucanase and mannanase activities, although they did not display cellulase or xylanase activity. 
This result is consistent with Noots et al. (34), who reported that $\beta$-glucan was intensively degraded by Mucor sp., although no cellulase or xylanase activity was detected (26). Three of the $9(33 \%)$ Mucor strains isolated here displayed protease activity, which is considerably lower than that found among Mucor sp. isolates (82\%) reported by Alves et al. (3). However, the P. crustosum and A. fumigatus isolates from SDC showed high cellulase and xylanase activities, and relatively high $\beta$-glucanase and mannanase activities. Our results are supported by Jahangeer et al. (26), who reported cellulase production by Penicillium $\mathrm{sp}$. and Aspergillus sp., while the production of xylanase by these fungi was demonstrated by Chávez et al. (13) and Gawande and Kamat (22), respectively. Furthermore, $\beta$-glucanase activity of Penicillium sp. and Aspergillus sp. was reported by Santos et al. (44) and Noots et al. (34), respectively. The results of the present study suggest that during the composting of sawdust, the degradation of cellulose and xylan is mainly due to Penicillium and Aspergillus, whereas the bioconversion of $\beta$-glucans and mannans is performed by Mucor and Aspergillus isolates. Therefore, Penicillium, Aspergillus, and Mucor appear to play a major role in the degradation of SDC.

Regarding the CRC isolates, Coniochaeta and Fusarium spp. showed low activity for the degradation of xylan, $\beta$ glucan, and mannan, and did not possess cellulase activity. Although all Coniochaeta isolates exhibited protease activity, few other fungal isolates possessed such activity, which may reflect the low protein content of the waste materials present in the CRC. In contrast, nearly all Trichoderma/ Hypocrea, Penicillium, and Aspergillus isolates exhibited high cellulase, xylanase, $\beta$-glucanase, and mannanase activities when grown on WBA medium. Trichoderma spp., which have been reported to produce cellulase (19), xylanase (1), $\beta$-glucanase (33), and mannanase (41), have been shown by Vitikainen et al. (55) to be the main industrial producers of cellulase and hemicellulase. In addition, de Siqueira et al. (19) reported the production of cellulase by Penicillium and Aspergillus spp. Taken together, these results indicate that Trichoderma/Hypocrea, Penicillium, and Aspergillus isolates play a predominant role in the degradation of lignocellulosic materials present in CRC.

Penicillium and Aspergillus were commonly found in SDC and $\mathrm{CRC}$, with isolates of both genera having high cellulolytic and hemicellulolytic activities. In particular, P. crustosum (SDCF5) and A. fumigatus (SDCF16) showed the highest cellulase and xylanase activities among SDC isolates, which suggests they play a significant role in the biodegradation of cellulose and hemicellulose. Among CRC isolates, $P$. verruculosum (CRCF 6) and T. harzianum/H. lixii (CRCF2 and CRCF16) were found to have the highest cellulolytic and hemicellulolytic activities, indicating their importance for the degradation of cellulose and hemicellulose during the composting of coffee residue.

Therefore, the results of the current study could be useful for determining the possible contribution of each characterized fungus in the degradation of lignocellulosic waste materials during the composting process.

The results revealed that Penicillium, Aspergillus, and Mucor isolates made a major contribution to the degradation of SDC, while Penicillium, Aspergillus, and Trichoderma isolates had a large role in the degradation of CRC. A number of isolates, such as P. crustosum (SDCF5), P. verruculosum (CRCF6), and T. harzianum/H. lixii (CRCF2 and CRCF16), displayed high cellulolytic and hemicellulolytic activities, and could be useful for improving biodegradation processes involving lignocellulosic waste materials.

\section{Acknowledgements}

We are extremely grateful to the Egyptian government for providing financial assistance to the first author.

\section{References}

1. Abdel-Sater, M.A., and A.H.M. El-Said. 2001. Xylan-decomposing fungi and xylanolytic activity in agricultural and industrial wastes. Int. Biodeterior. Biodegrad. 47:15-21.

2. Ainsworth, G.C., F.K. Sparrow, and A.S. Sussman (ed.). 1973. The Fungi: An Advanced Treatise, vol. 4A. Academic press, New York.

3. Alves, M.H., G.M. Campos-Takaki, A.L.F. Porto, and A.I. Milanez. 2002. Screening of Mucor spp. for the production of amylase, lipase, polygalacturonase and protease. Braz. J. Microbiol. 33:325-330.

4. Anastasi, A., G.C. Varese, and V.F. Marchisio. 2005. Isolation and identification of fungal communities in compost and vermicompost. Mycologia 97:33-44

5. Anderson, I.C., C.D. Campbell, and J.I. Prosser. 2003. Potential bias of fungal $18 \mathrm{~S}$ rDNA and internal transcribed spacer polymerase chain reaction primers for estimating fungal biodiversity in soil. Environ. Microbiol. 5:36-47.

6. Ashraf, R., F. Shahid, and T.A. Ali. 2007. Association of fungi, bacteria and actinomycetes with different composts. Pak. J. Bot. 39:2141-2151.

7. Aygan, A., and B. Arikan. 2008. A new halo-alkaliphilic, thermostable endoglucanase from moderately halophilic Bacillus sp. C14 isolated from van Soda lake. Int. J. Agric. Biol. 10:369-374.

8. Barnett, H.L., and B.B. Hunter. 1987. Illustrated Genera of Imperfect Fungi (4 ${ }^{\text {th }}$ edn.). Macmillan Publication Company, New York.

9. Béguin, P., and J.P. Aubert. 1994. The biological degradation of cellulose. FEMS Microbial. Rev. 13:25-58.

10. Bennett, J.W., P. Hollrah, A. Waterhouse, and K. Horvath. 1995. Isolation of bacteria and fungi from TNT-contaminated composts and preparation of ${ }^{14} \mathrm{C}$-ring labeled TNT. Int. Biodeterior. Biodegrad. 35:421-430.

11. Bonito, G., O.S. Isikhuemhen, and R. Vilgalys. 2010. Identification of fungi associated with municipal compost using DNA-based techniques. Bioresour. Technol. 101:1021-1027.

12. Brand, D., A. Pandey, S. Roussos, and C.R. Soccol. 2000. Biological detoxification of coffee husk by filamentous fungi using a solid state fermentation system. Enzyme Microb. Technol. 27:127-133.

13. Chàvez, R., P. Bull, and J. Eyzaguirre. 2006. The xylanolytic enzyme system from the genus Penicillium. J. Biotechnol. 123:413-433.

14. Chen, H., X. Li, and L.G. Ljungdahl. 1997. Sequencing of a 1,3-1,4$\beta \beta$-D-glucanase (lichenase) from the anaerobic fungus Orpinomyces strain PC-2: Properties of the enzyme expressed in Escherichia coli and evidence that the gene has a bacterial origin. J. Bacteriol. 179:6028-6034.

15. Cohen, R., and Y. Hadar. 2001. The roles of fungi in agricultural waste conversion, p. 305-334. In G.M. Gadd (ed.), Fungi in Bioremediation. Cambridge University Press, Cambridge.

16. Coman, G., M. Cotarlet, G. Bahrim, and P. Stougaard. 2008. Increasing the efficiency of screening streptomycetes able to produce glucanases by using insoluble chromogenic substrates. Roum. Biotechnol. Lett. 13: supplement, 20-25.

17. Damm, U., P.H. Fourie, and P.W. Crous. 2010. Coniochaeta (Lecythophora), Collophora gen. nov. and Phaeomoniella species associated with wood necroses of Prunus trees. Persoonia 24:60-80.

18. de Hoog, G.S., and M. Th. Smith. 2004. Ribosomal gene phylogeny and species delimitation in Geotrichum and its teleomorphs. Stud. Mycol. 50:489-515. 
19. de Siqueira, F.G., E.G. de Siqueira, P.M.D. Jaramillo, M.H.L. Silveira, J. Andreaus, F.A. Couto, L.R. Batista, and E.X.F. Filho. 2010. The potential of agro-industrial residues for production of holocellulase from filamentous fungi. Int. Biodeterior. Biodegrad. 64:20-26.

20. Decker, S.R., W.S. Adney, E. Jennings, T.B. Vinzant, and M.E. Himmel. 2003. Automated filter paper assay for determination of cellulase activity. Appl. Biochem. Biotechnol. 107:689-703.

21. Duedahl-Olesen, L., L.H. Pedersen, and K.L. Larsen. 2000. Suitability and limitations of methods for characterization of activity of malto-oligosaccharide-forming amylases. Carbohydr. Res. 329:109-119.

22. Gawande, P.V., and M.Y. Kamat. 2000. Production of xylanases by immobilized Aspergillus sp. using lignocellulosic waste. World J. Microbiol. Biotechnol. 16:111-112.

23. Hatamoto, M., T. Tanahashi, J. Murase, K. Matsuya, M. Hayashi, M. Kimura, and S. Asakawa. 2008. Eukaryotic communities associated with the decomposition of rice straw compost in a Japanese rice paddy field estimated by DGGE analysis. Biol. Fertil. Soils 44:527532 .

24. Hortin, G.L., I. Warshawsky, and M. Laude-Sharp. 2001. Macromolecular chromogenic substrates for measuring proteinase activity. Clin. Chem. 47:215-222.

25. Howard, R.L., E. Abotsi, E.L. Jansen van Rensburg, and S. Howard. 2003. Lignocellulose biotechnology: Issues of bioconversion and enzyme production. Afr. J. Biotechnol. 2:602-619.

26. Jahangeer, S., N. Khan, S. Jahangeer, M. Sohail, S. Shahzad, A. Ahmad, and S.A. Khan. 2005. Screening and characterization of fungal cellulases isolated from the native environmental source. Pak. J. Bot. 37:739-748.

27. Jasalavich, C.A., A. Ostrofsky, and J. Jellison. 2000. Detection and identification of decay fungi in spruce wood by restriction fragment length polymorphism analysis of amplified genes encoding rRNA. Appl. Environ. Microbiol. 66:4725-4734.

28. Jing-Chun, T., Q. Zhou, and A. Katayama. 2010. Effects of raw materials and bulking agents on the thermophilic composting process. J. Microbiol. Biotechnol. 20:925-934.

29. Kumar, R., S. Singh, and O.V. Singh. 2008. Bioconversion of lignocellulosic biomass: Biochemical and molecular perspectives. J. Ind. Microbiol. Biotechnol. 35:377-391.

30. Lievens, B., S. van Kerckhove, A. Justé, B.P.A. Cammue, O. Honnay, and H. Jacquemyn. 2010. From extensive clone libraries to comprehensive DNA arrays for the efficient and simultaneous detection and identification of orchid mycorrhizal fungi. J. Microbiol. Methods 80:76-85.

31. Lord, N.S., C.W. Kaplan, P. Shank, C.L. Kitts, and S.L. Elrod. 2002. Assessment of fungal diversity using terminal restriction fragment (TRF) pattern analysis: Comparison of $18 \mathrm{~S}$ and ITS ribosomal regions. FEMS Microbiol. Ecol. 42:327-337.

32. Michaelsen, A., F. Pinzari, K. Ripka, W. Lubitz, and G. Piñar. 2006. Application of molecular techniques for identification of fungal communities colonising paper material. Int. Biodeterior. Biodegrad. 58:133-141.

33. Murashima, K., T. Nishimura, Y. Nakamura, J. Koga, T. Moriya, N. Sumida, T. Yaguchi, and T. Kono. 2002. Purification and characterization of new endo-1,4- $\beta$-D-glucanases from Rhizopus oryzae. Enzyme Microb. Technol. 30:319-326.

34. Noots, I., V. Derycke, K. Cornelis, C. Michiels, J.A. Delcour, R. Delrue, J.D. Keersmaeker, and T. Coppens. 2001. Degradation of starchy endosperm cell walls in nongerminating sterilized barley by fungi. J. Agric. Food Chem. 49:975-981.

35. Ofomaja, A.E., and Y.S. Ho. 2008. Effect of temperatures and $\mathrm{pH}$ on methyl violet biosorption by Mansonia wood sawdust. Bioresour. Technol. 99:5411-5417.

36. Pečiulytė, D. 2007. Isolation of cellulolytic fungi from waste paper gradual recycling materials. Ekologija 53:11-18.

37. Pedersen, M., M. Hollensted, L. Lange, and B. Andersen. 2009. Screening for cellulose and hemicellulose degrading enzymes from the fungal genus Ulocladium. Int. Biodeterior. Biodegrad. 63:484489
38. Pérez, J., J. Muñoz-Dorado, T. de-la-Rubia, and J. Martínez. 2002. Biodegradation and biological treatments of cellulose, hemicellulose and lignin: An overview. Int. Microbiol. 5:53-63.

39. Pitt, J.I. 1994. The current role of Aspergillus and Penicillium in human and animal health. J. Med. Vet. Mycol. 32:17-32.

40. Purnomo, A.S., F. Koyama, T. Mori, and R. Kondo. 2010. DDT degradation potential of cattle manure compost. Chemosphere 80:619-624.

41. Sachslehner, A., B. Nidetzky, K.D. Kulbe, and D. Haltrich. 1998. Induction of mannanase, xylanase, and endoglucanase activities in Sclerotium rolfsii. Appl. Environ. Microbiol. 64:594-600.

42. Sánchez, C. 2009. Lignocellulosic residues: Biodegradation and bioconversion by fungi. Biotechnol. Adv. 27:185-194.

43. Sánchez, G., E.J. Olguín, and G. Mercado. 1999. Accelerated coffee pulp composting. Biodegradation 10:35-41.

44. Santos, T., J.R. Villanueva, and C. Nombela. 1977. Production and catabolite repression of Penicillium italicum $\beta$-glucanases. J. Bacteriol. 129:52-58.

45. Sınağ, A., S. Gülbay, B. Uskan, and M. Güllü. 2009. Comparative studies of intermediates produced from hydrothermal treatments of sawdust and cellulose. J. Supercrit. Fluids 50:121-127.

46. Singh, H. 2006. Mycoremediation: Fungal Bioremediation. John Wiley \& Sons, Hoboken, New Jersey.

47. Sukumaran, R.K., R.R. Singhania, and A. Pandey. 2005. Microbial cellulases-production, applications and challenges. J. Sci. Ind. Res. 64:832-844.

48. Tamura, K., J. Dudley, M. Nei, and S. Kumar. 2007. MEGA4: Molecular evolutionary genetics analysis (MEGA) software version 4.0. Mol. Biol. Evol. 24:1596-1599.

49. Teather, R.M., and P.J. Wood. 1982. Use of congo red-polysaccharide interactions in enumeration and characterization of cellulolytic bacteria from the bovine rumen. Appl. Environ. Microbiol. 43:777780.

50. Ten, L.N., W.T. Im, M.K. Kim., M.S. Kang, and S.T. Lee. 2004. Development of a plate technique for screening of polysaccharidedegrading microorganisms by using a mixture of insoluble chromogenic substrates. J. Microbiol. Methods 56:375-382.

51. Thompson, J.D., T.J. Gibson, F. Plewniak, F. Jeanmougin, and D.G. Higgins. 1997. The Clustal_X windows interface: Flexible strategies for multiple sequence alignment aided by quality analysis tools. Nucleic Acids Res. 25:4876-4882.

52. Udagawa, S., K. Tubaki, Y. Horie, K. Miura, K. Minoura, M. Yamazaki, T. Yokoyama, and S. Watanbe. 1978. Kinrui Zukan (An illustration book of the fungus). Kodansha, Tokyo (in Japanese).

53. Valášková, V., J. Šnajdr, B. Bittner, T. Cajthaml, V. Merhautová, M. Hofrichter, and P. Baldrian. 2007. Production of lignocellulosedegrading enzymes and degradation of leaf litter by saprotrophic basidiomycetes isolated from a Quercus petraea forest. Soil Biol. Biochem. 39:2651-2660.

54. Varnaite, R., and V. Raudonienè. 2008. Destruction of hemicellulose in rye straw by micromycetes. Ekologija 54:169-172.

55. Vitikainen, M., M. Arvas, T. Pakula, M. Oja, M. Penttilä, and M. Saloheimo. 2010. Array comparative genomic hybridization analysis of Trichoderma reesei strains with enhanced cellulase production properties. BMC Genomics 11:441.

56. Wen, Z., W. Liao, and S. Chen. 2005. Production of cellulase/ $\beta$ glucosidase by the mixed fungi culture Trichoderma reesei and Aspergillus phoenicis on dairy manure. Process Biochem. 40:30873094.

57. White, T.J., T. Bruns, S. Lee, and J. Taylor. 1990. Amplification and direct sequencing of fungal ribosomal RNA genes for phylogenetics, p. 315-322. In M.A. Innis, D.H. Gelfand, J.J. Sninsky, and T.J. White (ed.), PCR Protocols: A Guide to Methods and Applications. Academic Press, New York.

58. Zhang, Y.H.P., M.E. Himmel, and J.R. Mielenz. 2006. Outlook for cellulase improvement: Screening and selection strategies. Biotechnol. Adv. 24:452-481.

59. Zhang, Z., S. Schwartz, L. Wagner, and W. Miller. 2000. A greedy algorithm for aligning DNA sequences. J. Comput. Biol. 7:203-214. 